\title{
Comparison of end-diastolic versus end-systolic cardiac-computed tomography reconstruction interval in patient's prior to pulmonary vein isolation
}

\author{
Wieland Staab ${ }^{1,3^{*}}$, Sabrina Goth ${ }^{1}$, Christian Sohns ${ }^{2,3}$, Jan Martin Sohns ${ }^{1,3}$, Michael Steinmetz ${ }^{1,3}$, \\ Christina Unterberg Buchwald ${ }^{1,2,3}$, Andreas Schuster ${ }^{2,3}$, Johannes Tammo Kowallick', Martin Fasshauer ${ }^{1,3}$ \\ and Joachim Lotz ${ }^{1,3}$
}

\begin{abstract}
Purpose: Aim of the study was to investigate diagnostic accuracy of cardiac computed tomography angiography (CCTA) between left ventricular end-systolic (LVES) and left ventricular end-diastolic (LVED) cardiac phase for thrombus detection in patient's prior to pulmonary vein isolation (PVI).

Materials and methods: 182 consecutive Patients with drug refractory AF scheduled for PVI $(62.6 \%$ male, mean age $64.1 \pm 10.2$ years) underwent routine pre-procedural evaluation including transesophageal echocardiography (TEE) and CCTA for evaluation of left atrial (LA)/left atrial appendage (LAA) anatomy and thrombus formation. Qualitative and quantitative analysis (using aorta ascendens (AA)/LAA ratio) was performed. Measurements of the LA/LAA in LVES and LVED cardiac phase were obtained.
\end{abstract}

Results: End-systolic volumes (LA/LAA) measured in 30 patients without filling defects as control group and all 14 with filling defects of 182 patients were significantly larger $(p<0.01)$ than in end-diastolic phase. Qualitative analysis was inferior to quantitative analysis using LA/LAA ratio (<0.5; accuracy: 100\%, 88\%,100\%, 99\% vs 100\%). 5 out of 182 patients (2.7\%) showed thrombus formation of the LAA in CCTA confirmed by TEE and quantitative analysis. Intra/-interobserver variability was lower in end-systolic vs end-diastolic reconstruction interval.

Conclusion: For evaluating CCTA datasets in patients prior PVI, the LVES reconstruction interval is recommended due to significantly larger LA/LAA volumes and lower intra/- interobserver variability's.

Keywords: PVl; Thrombus; LAA; Volumes

\section{Introduction}

Atrial fibrillation (AF) is the most common sustained cardiac arrhythmia with a significant increase of morbidity and mortality due to its associated risk of thrombembolism. Pulmonary vein isolation (PVI) by percutaneous radiofrequency ablation has emerged as a therapeutic option. Actually it is recommended for symptomatic and drug refractory patients (Romero et al. 2013). In order to

\footnotetext{
* Correspondence: wieland.staab@med.uni-goettingen.de 'Department of Diagnostic and Interventional Radiology, Georg-August-University, Robert-Koch-Str. 40, 37075 Goettingen, Germany ${ }^{3}$ DZHK, Göttingen, Germany

Full list of author information is available at the end of the article
}

prevent thrombembolic events, it is mandatory to exclude left atrial (LA) and LA appendage (LAA) thrombus prior to PVI (Dorenkamp et al. 2011; Scherr et al. 2009; Hur et al. 2009). Transesophageal echocardiography (TEE) is considered the gold standard modality in detecting left atrial (LA)/LA appendage (LA/LAA) thrombi (Dorenkamp et al. 2011; Scherr et al. 2009). However, this is a semi-invasive procedure with rare but potential life-threatening complications (Scherr et al. 2009). Cardiac computed tomography angiography (CCTA) has been proposed as an alternative method (Romero et al. 2013; Dorenkamp et al. 2011; Scherr et al. 2009). Prior to PVI, CCTA and TEE are used to provide exact anatomical 
details of the size of the left atrium as well as the number and position of the pulmonary veins entering the left atrium and to exclude LA/LAA thrombus (Gage et al. 2004). Reported limitations of CCTA are the high false positive rate of thrombus formations and therefore low specificity, unacceptable interobserver variability's (Aljaroudi et al. 2013).

Prospective electrocardiography (ECG) triggering became available with newer scanner techniques, allowing data acquisition during a pre-defined, narrow portion of the $\mathrm{R}-\mathrm{R}$ interval resulting in a reduction in radiation dose (Steigner et al. 2009). Here, the mid-to-end-diastole at $70-75 \%$ of the R-R interval is mostly used for prospective-ECG- triggering, since coronary artery motion is small in that phase (Menke et al. 2013). In the traditional retrospective ECG-gated-CCTA method, the full cardiac cycle is imaged $(0-100 \%)$, and the heart is retrospectively reconstructed at the required cardiac phases (Dewey 2011). In this context, retrospective gating is chosen because temporal as well as dynamic information is obtained and CCTA images can be reconstructed at other cardiac phases $(0-100 \%)$, if the standard phase was not diagnostic or interpretable (Steigner et al. 2009; Menke et al. 2013). Therefore, we can choose appropriate phases for minimum and maximum LA/LAA diameters and volumes at end-diastole and end-systole for postprocessing and evaluating CCTA data. Aim of the study was to investigate diagnostic performance of thrombus detection in CCTA between cardiac cycles, intra/- interobserver variability's of thrombus detection as well as comparing acquired volumetric and diametric datasets.

\section{Materials and methods Patients}

182 consecutive prospectively evaluated patients with AF scheduled for PVI (62.6\% male, mean age $64.1 \pm 10.2$ years, Table 1) underwent routine diagnostic work up prior to PVI including TEE and CCTA (from January 2010 until May 2013). In all cases CCTA and TEE were performed within 1-3 days prior to PVI. The analysis was approved by the local ethic committee (University Medical Center Göttingen). The CHADS2 scoring system (Romero et al. 2013; Scherr et al. 2009; Camm et al. 2012) was used for risk stratification of thromboembolic events. The scoring system assigns one point for the presence of age $>75$, heart failure, diabetes mellitus and hypertension and two points for prior transitory ischemic attack (TIA) or stroke. Further risk factors of LA/LAA thrombus such as chronic kidney disease, valvular disease, cardiomyopathy and LA size were evaluated and documented (Camm et al. 2012), Table 2.

\section{Computed tomography}

CCTA was performed with a 64-slice MDCT scanner (VCT LightSpeed, GE Healthcare, Milwaukee, WI, USA),
Table 1 Patient demographic characteristics

\begin{tabular}{ll}
\hline Male & $114(62.6 \%)$ \\
Female & $68(37.4 \%)$ \\
Age, mean & $64.1 \pm 10.2$ \\
Age $\geq \mathbf{7 5}$ years & $15(8.2 \%)$ \\
Mean ejection fraction & $54.2 \pm 6.1$ \\
Paroxysmal atrial fibrillation & $119(65.4 \%)$ \\
Persistent atrial fibrillation & $63(34.6 \%)$ \\
Cardiomyopathy (dilated) & $9(4.9 \%)$ \\
Mitral/aortic valve disease & $29(15.9 \%)$ \\
Serum creatinine; mg/dl & $0.94 \pm 0.27$ \\
Elevated serum creatinine & $27(14.8 \%)$ \\
CHADS 2 score: & \\
CHADS2 $=\mathbf{0}$ & $41(22.5 \%)$ \\
CHADS2 $=\mathbf{1}$ & $92(50.5 \%)$ \\
CHADS2 $=\mathbf{2}$ & $36(19.8 \%)$ \\
CHADS2 $\geq \mathbf{3}$ & $13(7.1 \%)$ \\
\hline
\end{tabular}

slice collimation $64 \times 0.625 \mathrm{~mm}$; rotation time $600 \mathrm{msec}$; tube voltage $100-120 \mathrm{kV}$; adaptive dose regime (auto mAs, 280-380). Depending on the scan range and the patient's body weight the calculated mean radiation dose was $6.1 \pm 2.6 \mathrm{mSv}$ (dose-length product range, 137 to $537 \mathrm{mGy}$ * $\mathrm{cm}$ multiplied with the conversion coefficient for the chest of $\mathrm{k}=0.017 \mathrm{mSv} \mathrm{mGy}^{-1} \mathrm{~cm}^{-1}$ ). Retrospective ECG gated half scan algorithm was used to reconstruct the data into contiguous axial images with a slice thickness of $0.625 \mathrm{~mm}$. For this study, images were evaluated at end- diastolic as well as end-systolic cardiac cycle ( $39 \pm 4 \%$ and $77 \pm 5 \%$ of the RR-interval) on a dedicated workstation (Aquarius 3D Workstation, TeraRecon, San Mateo, CA, USA). Beta adrenergic blocking agents were administered prior the scan procedure if the heart rate was $>70 \mathrm{bpm}$ (79\% of the investigated patients) (mean heart rate $61 \pm 8 \mathrm{bpm}$ ). Contrast media injection protocol (split-bolus) was as followed: injection of $30 \mathrm{ml}$ at $2 \mathrm{ml} / \mathrm{sec}$ of iodinated intravenous contrast agent (Imeron 350, Bracco, Konstanz, Germany); a 20 sec break followed by $70 \mathrm{ml}$ at $4 \mathrm{ml} / \mathrm{sec}$ of the same contrast and a $40 \mathrm{ml}$ saline chaser at $4 \mathrm{ml} / \mathrm{sec}$ in a single spiral scan technique within a single breath-hold covering an area from the aortic arch to below the diaphragm. Semi-automatic bolus chasing was used to detect the second contrast bolus in the ascending aorta. CCTA images were analyzed separately at $39 \pm 4$ and $77 \pm 5 \%$ RR-interval (quantitative and qualitative analysis at end-diastolic as well as end-systolic cardiac cycle independently by both readers) blinded to TEE results by two experienced independent readers. Complete CCTA Datasets were evaluated by both readers independently at two different time periods. Qualitative, visual assessment 
Table 2 Clinical characteristics of patients with and without LA/LAA thrombus

\begin{tabular}{|c|c|c|c|}
\hline & $\begin{array}{l}\text { No LA/LAA } \\
\text { thrombus }\end{array}$ & $\begin{array}{l}\text { LA/LAA } \\
\text { thrombus }\end{array}$ & p-value \\
\hline & $\mathrm{N}=177(97.3 \%)$ & $\mathrm{N}=5(2.7 \%)$ & \\
\hline Male & $110(62.1 \%)$ & $4(80 \%)$ & n.s. \\
\hline Female & 67 (37.9\%) & $1(20 \%)$ & n.s. \\
\hline Age, mean & $64.0 \pm 8.8$ & $68.8 \pm 12.9$ & 0.01 \\
\hline Mean ejection fraction & $54.3 \pm 6.0$ & $48.6 \pm 7.7$ & n.s. \\
\hline Paroxysmal atrial fibrillation & $116(65.5 \%)$ & $3(60 \%)$ & n.s. \\
\hline Persistent atrial fibrillation & $61(34.5 \%)$ & $2(40 \%)$ & n.s. \\
\hline Cardiomyopathy (dilated) & $9(5.1 \%)$ & $0(0 \%)$ & n.s. \\
\hline Mitral/aortic valve disease & $27(15.3 \%)$ & $2(40 \%)$ & n.s. \\
\hline Serum creatinine; mg/dl & $0.93 \pm 0.27$ & $1.12 \pm 0.20$ & n.s. \\
\hline Elevated serum creatinine & $25(14.1 \%)$ & $2(40 \%)$ & n.s. \\
\hline \multicolumn{4}{|l|}{ CHADS 2} \\
\hline Congestive heart failure & $33(18.6 \%)$ & $1(20 \%)$ & n.s. \\
\hline Hypertension & $111(62.7 \%)$ & $4(80 \%)$ & n.s. \\
\hline Diabetes mellitus & $30(16.9 \%)$ & $4(80 \%)$ & 0.0004 \\
\hline Prior stroke or TIA & $7(4.0 \%)$ & $0(0 \%)$ & n.s. \\
\hline Age $\geq 75$ years & $12(6.8 \%)$ & $3(60 \%)$ & $<0.0001$ \\
\hline $\mathrm{CHADS} 2=0$ & $41(23.2 \%)$ & $0(0 \%)$ & n.s. \\
\hline $\mathrm{CHADS} 2=1$ & $92(52.0 \%)$ & $0(0 \%)$ & 0.009 \\
\hline $\mathrm{CHADS} 2=2$ & $33(18.6 \%)$ & $3(60 \%)$ & 0.02 \\
\hline CHADS2 $\geq 3$ & $11(6.2 \%)$ & $2(40 \%)$ & 0.004 \\
\hline
\end{tabular}

CHADS2: congestive heart failure, hypertension, age $>75$ years, diabetes and stroke (doubled).

of the LA/LAA was done using on out of three categories: no-thrombus, contrast filling defect or definite thrombus formation. A filling defect was defined as an intracavitary low attenuating round or oval lesion representing incomplete mixing of contrast agent and blood, whereas thrombus formation was defined as round-or oval shaped low attenuation (LAA/AA ratio $<0.5$ ) area (Romero et al. 2013; Hur et al. 2009; Hur et al. 2013). Quantitative measurement of relative contrast enhancement of the LA and LAA to the ascending aorta was done in all patients. Here, a $1 \mathrm{~cm}^{2}$ region of interest (ROI) was placed inside the filling defect in the LAA seen on CCTA images and the AA to generate the LAA/AA-ratio. In case no filling defect was observed in CCTA images, a $1 \mathrm{~cm}^{2}$ ROI was as well placed in the LAA and the AA for calculating LAA attenuation to AAo attenuation values. A cut-off value of LAA/AA ratio $<0.5$ was used to differentiate between thrombus and filling defect on CCTA.

\section{Echocardiography}

Transesophageal echocardiography (TEE) as well as transthoracic echocardioagraphy (TTE) was performed in all patients. TEE was obtained using a GE Vivid E9 ultrasound system (General Electric Ultrasound, Horten, Norway) with a $5.0 \mathrm{MHz}$ multiplane probe acquiring continuous cine loops of LA/LAA in $0^{\circ}-180^{\circ}$. TTE was used to determine left ventricular ejection fraction and LA size. Images were acquired according to the methods described by the American Society of Echocardiography from parasternal long and short axis, long axis, apical four chamber and two chamber views (Camm et al. 2012). Highly experienced (at least 4 years experience) cardiologists performed and interpreted all TEEs blinded to patient's history and results from other procedures such as CCTA. Thrombus was defined as a distinct intracavitary echo-lucent or echo-dense mass in comparison to dense non-clearing spontaneous echo contrast (SEC), defined as a slow swirling smoke like echo -density (Scherr et al. 2009; Hur et al. 2009; Hur et al. 2013). The teams of TEE/TTE and CCTA were blinded to the results of the other modality during the assessment of LA/LAA thrombus formation.

\section{Definitions and measurements of LA/LAA}

Reformats were done on a dedicated workstation (Aquarius 3D Workstation, TeraRecon ${ }^{\circ}$, San Mateo, CA, USA) (Figure 1). Minimal left atrial volume (LAV) was defined at left ventricular (LV) end-diastole (ED) when the LV was largest and LA smallest whereas maximum LAV was defined at LV end-systole (ES) directly before mitral valve opening when LAV was largest. The left atrial appendage (LAA) was evaluated separately. LAV was quantified according to a modified Simpson's method (Stojanovska et al. 2011). For quantifying LA diameters in sagittal, coronal and axial direction, reformatted images were obtained at the level of the aortic valve parallel to the LV outflow tract in LVES for maximum LA diameters and in LVED for minimum LA diameters. LAA was measured according to this method for minimal (LVED) and maximum (LVES) diameters and volumes. As a statistical significant homogenous group, 14 patients with detected thrombus or filling defect of LAA on CCTA (Table 2) were assessed and 30 patients without filling defects on CCTA 15 male (50\%), 15 with persistent AF (50\%), mean age $62.3 \pm 5.6$ were evaluated as control group. Intra/-intraobserver variability's for data reproducibility was independently obtained.

\section{Statistical analysis}

Biostatistics were planned and performed by the local Department of Medical Statistics. Descriptive statistical analyses, variables were expressed as the means \pm standard deviations and/or percentages. Intra/-interobserver agreement for LA/LAA thrombus was calculated using Cohen's kappa (к) statistics (kappa values: poor $<0.20$, fair 0.21-0.40, moderate $0.41-0.60$, good 0.61-0.80, excellent 0.81-1.00). Student's t-tests and Fisher's exact tests were used to 


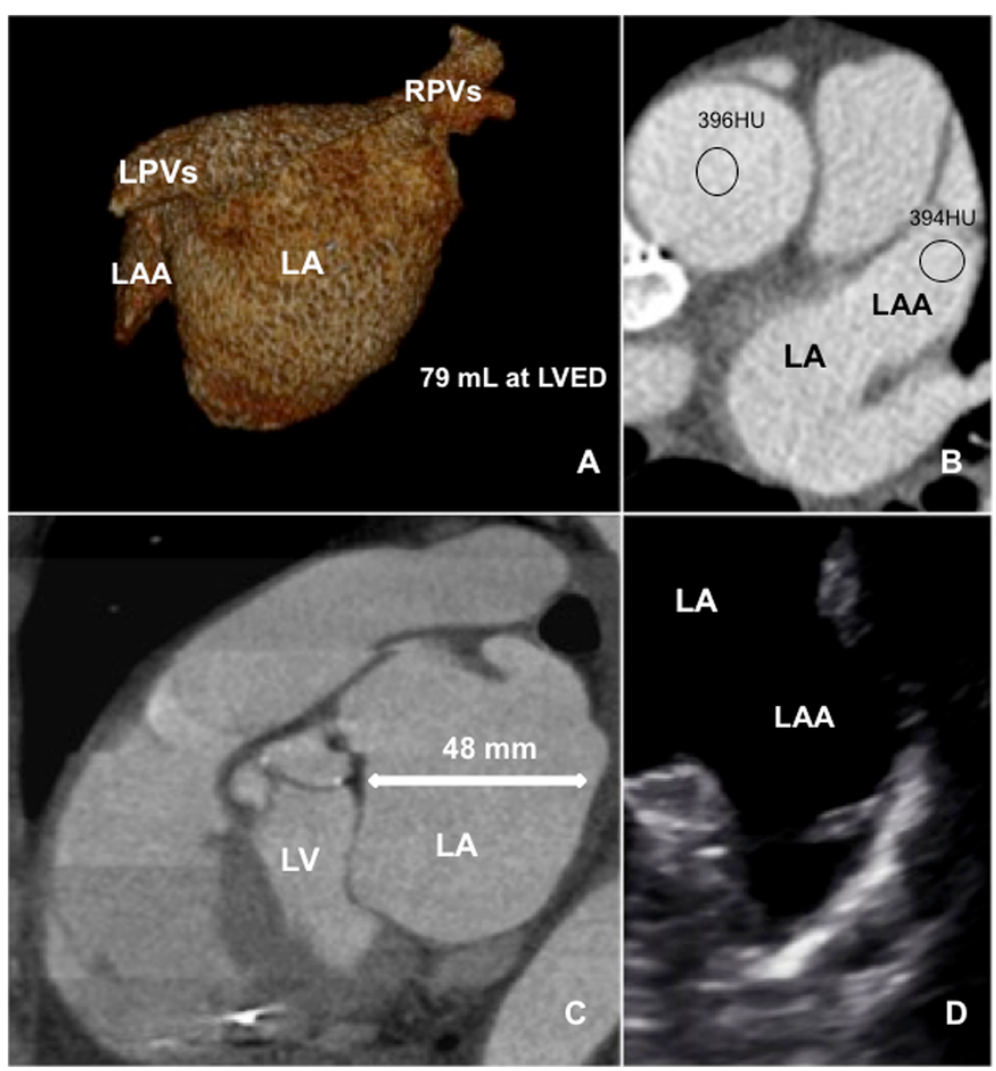

Figure 13 - Dimensional VR (A) with measured LA volume using the Simpsons method at LVED from a 71 year old female patient with paroxysmal AF prior PVI; CCTA (B) demonstrates trabeculated fully opacified LAA with LAA/AA ratio from 0.99; CCTA (C) reconstructed in oblique sagittal view showing LA at LVED on a dedicated workstation using TeraRecon $\odot$; Corresponding TEE (D) shows trabecularisation of the LAA without SEC or thrombus.

compare population averages and statistical significance of categorical population differences or Chi-squares test for eventually categorical variables for independence between groups. Sensitivity, specificity, negative (NPV) and positive predictive values (PPV) were assessed assuming TEE as reference standard for thrombus detection by using chi-squared test. For all obtained data, $\mathrm{p}<0.05$ was considered to be statistical significant. Statistical analysis was performed using SAS/STAT software (version 9.3, SAS Institute).

\section{Results}

\section{Patient characteristics}

Patient characteristics of all 182 consecutive patients are summarized in Table 1. Image quality of all 182 consecutive CCTA examinations was regarded to be diagnostic. Left atrial and left atrial appendage (LA/LAA) evaluation was feasible in all cases and no adverse events were reported during TEE and CCTA examination. CCTA and TEE examinations were performed within 3 days ( 2 days \pm 1 ). CCTA showed 14 out of 182 patients with filling defects in the LAA. Using LAA/AAo ratio of 0.5 , five patients showed definite thrombus formation, confirmed by TEE. These patients were excluded from ablation procedure. Paroxysmal AF was present in $65.4 \%$ of patients whereas $34.6 \%$ showed persistent AF. $78 \%$ of patients were treated with phenprocumon at time of CCTA and TEE exam, 17\% used aspirin for anticoagulation. At time of CCTA, AF was present in 61 patients (33.5\%).

\section{Thrombus formation in CCTA versus TEE and related characteristics}

In 5 cases a definitive thrombus formation using quantitative analysis (cut-off value of LA/LAA coefficient $<0.5$ ) was seen in the LAA confirmed by TEE (Table 2). 9 patients (3 with paroxysmal AF) showed filling defects without definitive thrombus formation using quantitative analysis (cut-off value of LA/LAA coefficient $>0.5$, Figure 2) judged as thrombotic precursors in the state of circulatory stasis confirmed by TEE (all patients showed slow-flow/ SEC of the LAA). TEE identified 2 additional patients with SEC in the LA or LAA that were missed by CCTA. Patients with detected LAA thrombus (5 out of 182, 2.7\%, Figure 3) showed absolutely a lower mean ejection fraction $(54.3 \pm 6.0 \%$ vs $48.6 \pm 7.7 \%)$, a higher serum creatinine $(\mathrm{mg} / \mathrm{dl})(0.93 \pm 0.27$ vs $1.12 \pm 0.2)$ and relatively more 


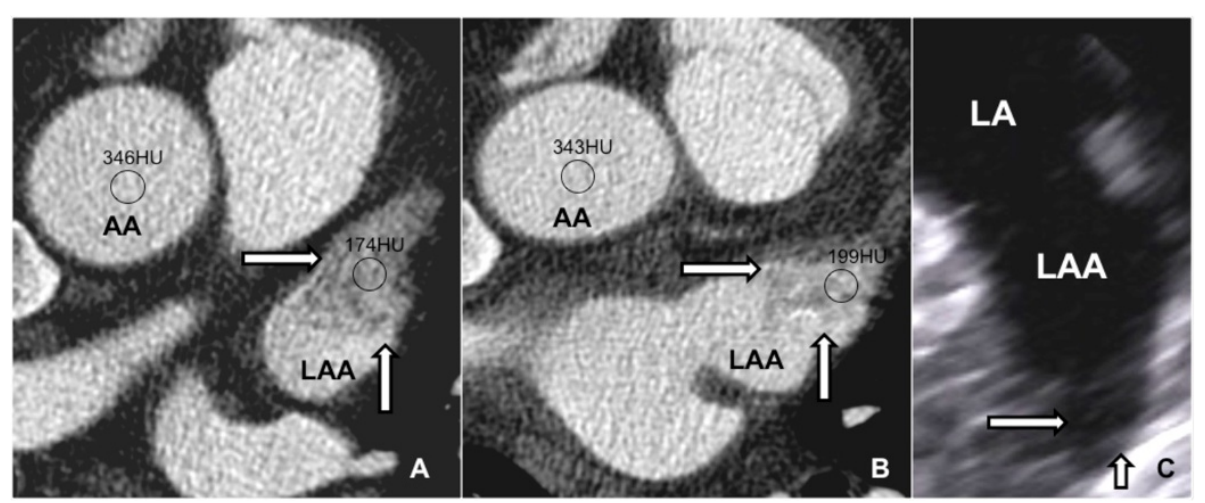

Figure 2 CCTA with filling defect (arrows) at LVED (A) and LVES (B); LAA is visually and quantitatively better opacified at LVES (A) (LAA/AA ratio 0.51 (A) and 0.59 (B)) indicating SEC, excluding thrombus, confirmed by TEE (C) (arrows) in a 63 year old male patient prior PVI with persistent AF.

patients with mitral or aortic valve disease (40\% vs $15.3 \%)$. In concordance to the CHADS2 score, diabetes mellitus $(16.9 \%$ vs $80 \%$, p value 0.0004$)$ and age $\geq 75$ (6.8\% vs $60 \%$, p value $<0.0001$ ) were significantly more prevalent in patients with LAA thrombus. Significantly higher CHADS2 scores were present in patients with thrombus (CHADS $2=2 ; 18.6 \%$ vs $60 \%$, p value 0.02 and CHADS $2 \geq 3 ; 6.2 \%$ vs $40 \%$, p value 0.004 ). LA/LAA thrombus was not detected in patients with a CHADS2 score $\leq 1$ and an age $\leq 50$ years.

\section{Measurements of LA and LAA}

In 30 patients without thrombus, diameters as well as volumes (absolute and indexed) were significantly larger in the LVES at $39 \pm 4 \%$ RR-interval (Table 3) $(\mathrm{p}<0.01)$. In 14 patients with filling defect on CCTA, diameters and volumes as well as indexed values were significantly larger in the LVES at $39 \pm 4 \%$ RR-interval $(\mathrm{p}<0.01)$ than in LVED. Patients with thrombus showed larger diameters and volumes as well as indexed values than patients without $(\mathrm{p}<0.01)$. Measurements were done blinded to patient's data 2 times within 6 weeks.

\section{Intra-/Interobserver variability's}

Assuming TEE as standard of reference for detection of thrombus or SEC, qualitative visual CCTA analysis at $77 \pm 5 \%$ of RR-interval resulted in a sensitivity of $94 \%$, specificity of $84 \%$, a negative predictive value (NPV) of $92 \%$ and a positive predictive value (PPV) of $87 \%$ whereas visual evaluation at $39 \pm 4 \%$ RR-interval showed superior results of $100 \%$ sensitivity, $94 \%$ specificity, $100 \%$ NPV and 93\% PPV for both readers in consensus. Applying a value of $<0.5$ (LAA/AA ratio) relative contrast enhancement in CCTA as a threshold of thrombus-formation, the overall sensitivity, specificity, NPV and PPV were 100\% independently for both readers at both dates at $39 \pm 4$ and $77 \pm 5 \%$ RR-Interval. Intra/-interobserver variability's were lower at absolute values at LVES in both readers $(39 \pm 4 \%$ RR-interval; $\kappa=0.942$ vs 0.901$)$ vs LVED $(77 \pm 5 \%$ RR-interval; $\mathrm{K}=0.891$ vs 0.860 ).

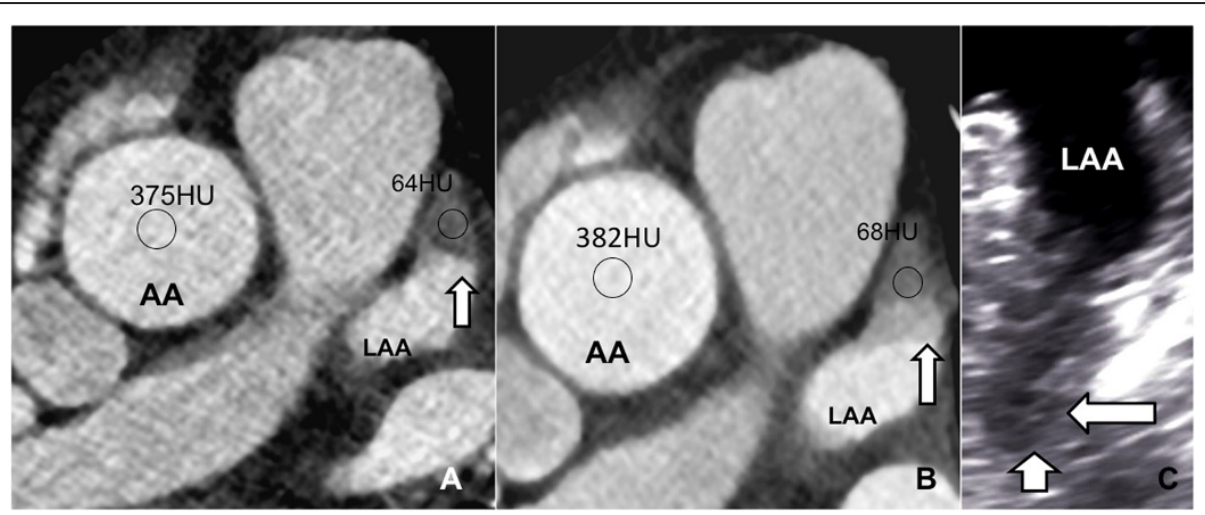

Figure 3 CCTA with depicted filling defect (arrows) in the LAA at LVED (A) and LVES (B) showing thrombus formation (LAA/AA ratio 0.17 (A) vs 0.18 (B)) in a 64 year old male patient with persistent AF confirmed by TEE (C). Additional visible SEC on top of thrombus with higher density at LVES (B) and TEE. 
Table 3 Measurements including diameters, volumes and indexed values of diameters and volumes showing significant differences in volumes and diameters between LVED und LVES

\begin{tabular}{|c|c|c|c|c|c|c|}
\hline Measurement & $\begin{array}{l}30 \text { Patients without } \\
\text { filling defect at LVED }\end{array}$ & $\begin{array}{l}30 \text { Patients without } \\
\text { filling defect at LVES }\end{array}$ & p-value & $\begin{array}{l}14 \text { Patients with } \\
\text { filling defect at LVED }\end{array}$ & $\begin{array}{l}14 \text { Patients with } \\
\text { filling defect at LVES }\end{array}$ & p-value \\
\hline Diameter- axial (mm) & $75.90+/-7.95$ & $87.10+/-8.81$ & $<0.01$ & $80.25+/-7.74$ & $88.85+/-8.45$ & $<0.01$ \\
\hline Diameter - sagittal (mm) & $38.25+/-6.6$ & $53.10+/-7.23$ & $<0.01$ & $46.70+/-5.45$ & $54.50+/-6,77$ & $<0.01$ \\
\hline Diameter - coronar (mm) & $74.05+/-9.3$ & $79.90+/-5,16$ & $<0.01$ & $78.70+/-9.32$ & $85.50+/-6.72$ & $<0.01$ \\
\hline Volume- $\mathrm{LA}(\mathrm{mL})$ & $169.50+/-31.62$ & $176.51+/-34.57$ & $<0.01$ & $187.00+/-32.87$ & $192.5+/-33.59$ & 0.026 \\
\hline Volume- LAA (mL) & 6.673 .45 & $9.39+/-4.87$ & $<0.01$ & $8.71+/-2.24$ & $10.72+/-3.55$ & 0.03 \\
\hline Diameter- axial/BSA $\left(\mathrm{mm} / \mathrm{m}^{2}\right)$ & $37.64+/-7.23$ & $41.78+/-6.56$ & $<0.01$ & $38,83+/-7.99$ & $42.56+/-6.43$ & $<0.01$ \\
\hline Diameter - sagittal/BSA $\left(\mathrm{mm} / \mathrm{m}^{2}\right)$ & $19.27+/-4.08$ & $26.33+/-6.20$ & $<0.01$ & $22.82+/-5.79$ & $26.18+/-7.58$ & $<0.01$ \\
\hline Diameter - coronar/BSA $\left(\mathrm{mm} / \mathrm{m}^{2}\right)$ & $35.87+/-6.06$ & $40.16+/-7.53$ & $<0.01$ & $37.69+/-8.01$ & $40.941+/-8.99$ & $<0.01$ \\
\hline Volume- LA/BSA (mL/m²) & $82.00+/-21.68$ & $85,80+/-21.82$ & 0.04 & $90.66+/-23.58$ & $93.65+/-23.66$ & 0.02 \\
\hline Volume- LAA/BSA $\left(\mathrm{mL} / \mathrm{m}^{2}\right)$ & $3.2+/-1.82$ & $4.41+/-2.23$ & 0.035 & $4.14+/-3.21$ & $5.18+/-2.87$ & 0.25 \\
\hline
\end{tabular}

\section{Discussion}

Main finding

Quantitative analysis (threshold of $<0.5$ when using LAA/AA ratio) of CCTA filling defects is superior to qualitative visual analysis in detecting or ruling out LA/LAA thrombus. For evaluating CCTA datasets in patients prior to PVI, the LVES (at $39 \pm 4 \%$ RR-interval) reconstruction interval is recommended due to significantly larger LA/LAA diameters/volumes $(\mathrm{p}<0.01)$ and lower intra/- interobserver variability's $(\kappa=0.942$ vs $\kappa=0.891)$ in absolute values.

\section{Thrombus formation}

To minimize the risk of periprocedural thromboembolic events during or after catheter ablation in the LA, it is necessary to rule out LA/LAA thrombus formation prior to PVI. TEE is an effective, reproducible method for excluding intracavitary thrombi and actually remains the gold-standard to assess LA/LAA thrombus formation but sedation due to the invasiveness of the modality (Camm et al. 2012). Various study protocols for detection of LA/LAA thrombus have been described and discussed in the past few years (Dorenkamp et al. 2011; Hur et al. 2009; Aljaroudi et al. 2013; Hur et al. 2013). Commonly iodinated contrast agent is used in a single-bolus injection with monophasic or biphasic (early/late phase imaging) CT protocols (Dorenkamp et al. 2011; Hur et al. 2009; Aljaroudi et al. 2013). A major limitation of CCTA is incomplete contrast filling of the LAA, representing the most frequent cause of false positive filling defects, leading to a low PPV up to 30\% (Hur et al. 2009; Aljaroudi et al. 2013). Wazni et al. (Wazni et al. 2007) reported no increase of peri-procedural stroke if SEC was identified in TEE. Additionally, even appropriate anticoagulation did not have an influence on presence of SEC (Wazni et al. 2007). In the present study, 9 out of 11 patients with SEC in TEE had non-thrombotic filling defects in CCTA in the
LAA with a LAA/AA ratio $>0.5$. The frequency of $2.7 \%$ intracardiac thrombus formations prior PVI is in line with previous studies (Scherr et al. 2009). Here, pre-saturation of the blood pool using a split-bolus injection single-scan protocol seems to decrease non-thrombotic filling defects by increasing contrast filling of the LAA and thus improving PPV. CCTA is not only able to evaluate LA/LAA region, pulmonary veins but can as well identify coronary artery placque and calcification with high sensitivity and specificity for diagnosing coronary artery disease (CAD) in patients with AF prior to PVI (Sohns et al. 2012).

\section{Measurements of LA and LAA}

Evaluation of LA volume and diameter is crucial prior to PVI to adequately assess patients eligible for the procedure as well as after ablation procedure to observe or exclude post-procedural complications (Hur et al. 2009; Stojanovska et al. 2011). Larger LA volumes and/or diameters due to AF and LA/LAA thrombi are associated with an increased risk of stroke and other embolic events (Hur et al. 2009; Gage et al. 2004; Stojanovska et al. 2011). The American Society of Echocardiography and the European Association for Echocardiography proposed the Simpsons method or the biplane area length method for evaluation of LA volume and diameter (Calkins et al. 2012). The Simpsons method is recommended while it does not rely on geometric assumptions and LAA as well as the ostia of the pulmonary veins are excluded from analysis (Christiaens et al. 2009). Absolute as well as indexed values of mean LA volume and diameter for all ages were in line with previous published echocardiographic, computed tomography and cardiac MRI data (Stojanovska et al. 2011; Christiaens et al. 2009; Mahabadi et al. 2009; Hudsmith et al. 2007) when the exact method of analysis was applied. Main differences to the study were the method to evaluate the LA and LAA. Here, Hudsmith et al. (Hudsmith et al. 2007), who used 
cardiac MRI, included the LA appendage in the analysis, which therefore showed probably higher values. LA and LAA volumes and diameters are in line with those previously published (Stojanovska et al. 2011; Hudsmith et al. 2007). Guidelines from the American Society of Echocardiography have recommended LAV measurement to be used for LA size quantification in clinical practice (Calkins et al. 2012). As shown in previous studies (Stojanovska et al. 2011; Mahabadi et al. 2009; Hudsmith et al. 2007; Martinez 2009) LA diameter and volume are significantly influenced by BSA which indicates the need and appropriateness of indexing LA volume and diameter to BSA for reporting these parameters. Here, LAA volume and diameters were evaluated separately. Therefore, we evaluated absolute as well as indexed values in patients with paroxysmal and persistent AF as well as in patients with SEC and thrombus, showing significantly larger volumes and diameters in the LVES. However, the absolute as well as indexed values reported in this study are in line with those reported in previous published studies (Stojanovska et al. 2011; Christiaens et al. 2009; Mahabadi et al. 2009; Hudsmith et al. 2007; Martinez 2009). Intra/-interobserver variability's were lower at LVES (39 $\pm 4 \%$ RR-interval; $\kappa=0.942)$ vs LVED $(77 \pm 5 \%$ RR-interval; $\kappa=0.891)$ in absolute values, it can be assumed due to larger LA/LAA volumes and diameters $(\mathrm{p}<0.01)$ at LVES cardiac cycle.

\section{Prospective vs retrospective gating}

In CCTA, prospective ECG-triggering needs overall less radiation dose than retrospective ECG-gating but serves less cardiac phases for evaluation (Menke et al. 2013).

Retrospective gating can be used with tube current modulation, where the full tube dose is applied e.g. at $30-70 \%$ of the RR-interval and is (prospectively ECG-based) reduced otherwise. Prospective gating is usable with temporal padding where more than a $180^{\circ}$ half-scan is needed, e.g. to image the $70-80 \%$ RR-interval and to reconstruct the heart at 5\% steps afterwards. The target of the exposure can therefore be set at $40 \%$ of the RR-interval (left atrium maximal volume) on a prospective trigger approach (Menke et al. 2013; Tsiflikas et al. 2010). Retrospective gating is preferable in patients with tachyarrhythmia, because CCTA images can be reconstructed at the required cardiac phase if the standard phase (LVED; 75\% of RR-interval) was nonevaluatable (Menke et al. 2013). Prospective triggering is most appropriate in patients with regular normal heart rates and a maximum heart rate of about 75 beats $/ \mathrm{min}$. Discussing the general rules of radiation protection, at least in patients without tachyarrhythmia; CCTA could be performed with a prospectively triggered low-dose technique. Prospectively triggered CCTA should not be inferior to the more radiation-intense retrospectively gated method regarding the need for having reliable diagnostic information. Using a dual-source CT, all typical referral patients are mostly eligible for prospectively triggered CCTA due to the much faster image acquisition (e.g., $83 \mathrm{~ms}$ ) while using $2 \mathrm{x}$-ray sources (Menke et al. 2013; Tsiflikas et al. 2010). However, this may not be suitable in patients with tachyarrhythmia, where even retrospectively gated CCTA with dual-source CT may be challenging (Sun et al. 2011).

\section{Limitations}

In this study, we did not perform TEE or cardiac MRI measurements on the same subjects to compare the results with our LA/LAA diameters and volumes, but the results correlate with previously published LA/LAA diameters and values for MRI and TEE (Scherr et al. 2009; Stojanovska et al. 2011; Christiaens et al. 2009; Mahabadi et al. 2009; Hudsmith et al. 2007; Martinez 2009) obtained with comparable method of analysis. Previous studies (Stojanovska et al. 2011; Christiaens et al. 2009) have indicated that enlargement of the LA body is often not symmetric and that the antero-posterior LA linear dimension measurement may not be the most accurate and should not be used for risk stratification. Here, LA volume measurement is more reproducible and accurate for estimation of LA enlargement and may be predictive of cardiovascular outcome (Stojanovska et al. 2011). Physiologically, it can be assumed that LVES cardiac phase shows larger diameters in comparison to the LVED cardiac phase, therefore we performed measurements on 44 patients in this study as control group to proof this. TEE was considered the reference standard for thrombus and SEC detection. The absence or presence of LA thrombus was not confirmed by direct visual inspection of anatomic or surgical specimen. Despite that, TEE and CCTA were not performed the same day. However initial experience with a dual-source CT (DSCT) at our institution using the described contrast injection-protocol with a prospective trigger approach at LVES $(39 \pm 4 \%$ RR-interval) looks promising while significantly reducing radiation exposure to about $<1-3 \mathrm{mSv}$.

\section{Conclusion}

This is the first study to evaluate LA/LAA volumes and sizes in context of thrombus detection in patients prior to PVI at LVES and LVED cardiac cycle $(39 \pm 4 \%$ and $77 \pm 5 \%$ RR-interval). Here the LVES (at $39 \pm 4 \%$ RR-interval) reconstruction interval is recommended due to significantly larger LA/LAA diameters/volumes $(\mathrm{p}<0.01)$ and lower intra/- interobserver variability's ( $\mathrm{K}=0.942$ vs $\mathrm{K}=0.891$ ). Quantitative analysis (threshold of $<0.5$ when using LAA/AA ratio) of CCTA filling defects is superior to qualitative visual analysis in detecting or ruling out LA/LAA thrombus. 


\section{Competing interests}

None of the authors had financial and personal relationships with other people or organizations that could inappropriately influence their work.

\section{Authors' contributions}

All authors read and approved the final manuscript.

\section{Author details}

${ }^{1}$ Department of Diagnostic and Interventional Radiology,

Georg-August-University, Robert-Koch-Str. 40, 37075 Goettingen, Germany.

${ }^{2}$ Department of Cardiology and Pneumology, Georg-August-University,

Goettingen, Germany. 'ZZHK, Göttingen, Germany.

Received: 16 April 2014 Accepted: 23 April 2014

Published: 1 May 2014

\section{References}

Aljaroudi WA, Saliba WS, Wazni OM, Jaber WA (2013) Role of cardiac computed tomography and cardiovascular magnetic resonance imaging in guiding management and treatment of patients with atrial fibrillation: state of the art review. J Nucl Cardiol 20:426-442

Calkins H, Kuck KH, Cappato R, Brugada J, Camm AJ, Chen SA, Crijns HJ, Damiano RJ, Jr, Davies DW, DiMarco J, Edgerton J, Ellenbogen K, Ezekowitz MD, Haines DE, Haissaguerre M, Hindricks G, lesaka Y, Jackman W, Jalife J, Jais P, Kalman J, Keane D, Kim YH, Kirchhof P, Klein G, Kottkamp H, Kumagai K, Lindsay BD, Mansour M, Marchlinski FE, et al. (2012) HRS/EHRA/ECAS expert consensus statement on catheter and surgical ablation of atrial fibrillation: recommendations for patient selection, procedural techniques, patient management and follow-up, definitions, endpoints, and research trial design: a report of the Heart Rhythm Society (HRS) Task Force on Catheter and Surgical Ablation of Atrial Fibrillation (2012) Developed in partnership with the European Heart Rhythm Association (EHRA), a registered branch of the European Society of Cardiology (ESC) and the European Cardiac Arrhythmia Society (ECAS); and in collaboration with the American College of Cardiology (ACC), American Heart Association (AHA), the Asia Pacific Heart Rhythm Society (APHRS), and the Society of Thoracic Surgeons (STS). Endorsed by the governing bodies of the American College of Cardiology Foundation, the American Heart Association, the European Cardiac Arrhythmia Society, the European Heart Rhythm Association, the Society of Thoracic Surgeons, the Asia Pacific Heart Rhythm Society, and the Heart Rhythm Society. Heart Rhythm 9:632-696

Camm AJ, Lip GY, De Caterina R, Savelieva I, Atar D, Hohnloser SH, Hindricks G, Kirchhof P (2012) 2012 focused update of the ESC Guidelines for the management of atrial fibrillation: an update of the 2010 ESC Guidelines for the management of atrial fibrillation. Developed with the special contribution of the European Heart Rhythm Association. Eur Heart J 33:2719-2747

Christiaens L, Lequeux B, Ardilouze P, Ragot S, Mergy J, Herpin D, Bonnet B, Allal J (2009) A new method for measurement of left atrial volumes using 64-slice spiral computed tomography: comparison with two-dimensional echocardiographic techniques. Int J Cardiol 131:217-224

Dewey M (2011) Coronary CT versus MR angiography: pro CT-the role of CT angiography. Radiology 258:329-339

Dorenkamp M, Sohns C, Vollmann D, Lüthje L, Seegers J, Wachter R, Puls M, Staab W, Lotz J, Zabel M (2011) Detection of left atrial thrombus during routine diagnostic work-up prior to pulmonary vein isolation for atrial fibrillation: role of transesophageal echocardiography and multidetector computed tomography. Int J Cardiol 163:681-684

Gage BF, van Walraven C, Pearce L, Hart RG, Koudstaal PJ, Boode BS, Petersen P (2004) Selecting patients with atrial fibrillation for anticoagulation: stroke risk stratification in patients taking aspirin. Circulation 110:2287-2292

Hudsmith LE, Cheng AS, Tyler DJ, Shirodaria C, Lee J, Petersen SE, Francis JM, Clarke K, Robson MD, Neubauer S (2007) Assessment of left atrial volumes at 1.5 Tesla and 3 Tesla using FLASH and SSFP cine imaging. J Cardiovasc Magn Reson 9:673-679

Hur J, Kim YJ, Lee HJ, Ha JW, Heo JH, Choi EY, Shim CY, Kim TH, Nam JE, Choe KO, Choi BW (2009) Left atrial appendage thrombi in stroke patients: detection with two-phase cardiac CT angiography versus transesophageal echocardiography. Radiology 251:683-690

Hur J, Pak HN, Kim YJ, Lee HJ, Chang HJ, Hong YJ, Choi BW (2013) Dual-enhancement cardiac computed tomography for assessing left atrial thrombus and pulmonary veins before radiofrequency catheter ablation for atrial fibrillation. Am J Cardiol 112:238-244
Mahabadi AA, Samy B, Seneviratne SK, Toepker MH, Bamberg F, Hoffmann U, Truong U (2009) Quantitative assessment of left atrial volume by electrocardiographic-gated contrast-enhanced multidetector computed tomography. J Cardiovasc Comput Tomogr 3:80-87

Martinez MW (2009) Onward and upward! From ventricle to atria, volumetric measurements for chamber quantification. J Cardiovasc Comput Tomogr 3:88-89

Menke J, Unterberg-Buchwald C, Staab W, Sohns JM, Seif Amir Hosseini A, Schwarz A (2013) Head-to-head comparison of prospectively triggered vs retrospectively gated coronary computed tomography angiography: meta-analysis of diagnostic accuracy, image quality, and radiation dose. Am Heart J 165:154-163

Romero J, Husain SA, Kelesidis I, Sanz J, Medina HM, Garcia MJ (2013) Detection of left atrial appendage thrombus by cardiac computed tomography in patients with atrial fibrillation: a meta-analysis. Circ Cardiovasc Imaging 6:185-194

Scherr D, Dalal D, Chilukuri K, Dong J, Spragg D, Henrikson CA, Nazarian S, Cheng A, Berger RD, Abraham TP, Calkins H, Marine JE (2009) Incidence and predictors of left atrial thrombus prior to catheter ablation of atrial fibrillation. J Cardiovasc Electrophysiol 20:379-384

Sohns C, Kruse S, Vollmann D, Lüthje L, Dorenkamp M, Seegers J, Jacobshagen C, Leber AW, Obenauer S, Hasenfuss G, Zabel M (2012) Accuracy of 64multidetector computed tomography coronary angiography in patients with symptomatic atrial fibrillation prior to pulmonary vein isolation. Eur Heart J Cardiovasc Imaging 13:263-270

Steigner ML, Otero HJ, Cai T, Mitsouras D, Nallamshetty L, Whitmore AG, Ersoy H, Levit NA, Di Carli MF, Rybicki FJ (2009) Narrowing the phase window width in prospectively ECG-gated single heart beat 320-detector row coronary $C T$ angiography. Int J Cardiovasc Imaging 25:85-90

Stojanovska J, Cronin P, Patel S, Gross BH, Oral H, Chughtai K, Kazerooni EA (2011) Reference normal absolute and indexed values from ECG-gated MDCT: left atrial volume, function, and diameter. AJR Am J Roentgenol 197:631-637

Sun ML, Lu B, Wu RZ, Johnson L, Han L, Liu G, Yu FF, Hou ZH, Gao Y, Wang HY, Jiang S, Yang YJ, Qiao SB (2011) Diagnostic accuracy of dual-source CT coronary angiography with prospective ECG-triggering on different heart rate patients. Eur Radiol 21:1635-1642

Tsiflikas I, Drosch T, Brodoefel H, Thomas C, Reimann A, Till A, Nittka D, Kopp AF, Schroeder S, Heuschmid M, Burgstahler C (2010) Diagnostic accuracy and image quality of cardiac dual-source computed tomography in patients with arrhythmia. Int J Cardiol 143:79-85

Wazni OM, Beheiry S, Fahmy T, Barrett C, Hao S, Patel D, Di Biase L, Martin DO, Kanj M, Arruda M, Cummings J, Schweikert R, Saliba W, Natale A (2007) Atrial fibrillation in patients with therapeutic international normalized ratio: comparison of strategies of anticoagulation management in the periprocedural period. Circulation 116:2531-2534

doi:10.1186/2193-1801-3-218

Cite this article as: Staab et al:: Comparison of end-diastolic versus end-systolic cardiac-computed tomography reconstruction interval in patient's prior to pulmonary vein isolation. SpringerPlus 2014 3:218.

\section{Submit your manuscript to a SpringerOpen ${ }^{\odot}$ journal and benefit from:}

- Convenient online submission

- Rigorous peer review

- Immediate publication on acceptance

- Open access: articles freely available online

- High visibility within the field

- Retaining the copyright to your article

Submit your next manuscript at springeropen.com 